\title{
Außenpolitik unter Obama - pragmatischer Multilateralismus und transatlantische Annäherungen
}

\author{
Stefan Fröhlich*
}

\section{Überzogene Erwartungen in Europa}

Mit dem deutlichen Sieg von Barack Obama bei den amerikanischen Präsidentschaftswahlen, den Politiker wie die internationale Presse zu Recht als in jeder Hinsicht , historisch bewertet haben, um die enorme Bedeutung dieses Wahlsiegs für Amerika und den Rest der Welt zu beschreiben, geht eine Ära zu Ende, deren Bilanz verheerend ist: George W. Bush übergibt das wohl wichtigste politische Amt in der Welt an seinen Nachfolger, da die USA wie eine Weltmacht im Niedergang wirken. Politisch und militärisch, aber auch - für das amerikanische Volk viel dramatischer - moralisch sind die USA im Begriff ihre Führungsrolle zu verspielen. Dass in das Weiße Haus zumal ein ,African American' einzieht, macht den Führungswechsel in Washington gleich im doppelten Sinn zu einer , historischen ‘ Zäsur. Nicht nur hat der 4. November 2008 gezeigt, dass es den , amerikanischen Traum` auch im 21. Jahrhundert noch gibt; Obama selbst hat die Kraft dieses zukunftsgewandten Optimismus im Wahlkampf immer wieder beschworen (,Yes, we can“). Gleichzeitig drückte die enorme Mobilisierung des Wählerinteresses im Lande, die ihre Entsprechung im weltweiten Widerhall auf diese Wahl fand, die ungebrochene Vitalität des amerikanischen Gemeinwesens aus. Entgegen allen Abgesängen auf das Land, wonach dieses gerade auch sein größtes Plus verliere - nämlich seinen Zukunftsoptimismus - zeigte sich einmal mehr, dass die USA gerade dann zu Aufbruchstimmung und Begeisterungsfähigkeit fähig sind, wenn so viele das Land auf dem falschen Weg sehen. Und eben die Tatsache, dass derjenige, der das Land aus seiner vielleicht größten Wirtschaftskrise seit dem Ende der großen Depression und zwei bedrückenden Kriegen führen soll, ein vergleichsweise unerfahrener Senator ist, zeigt den Mut und die bedingungslose Bereitschaft zum viel zitierten Wandel.

Vor diesem Hintergrund ist der Erwartungsdruck, der auf dem neuen Präsidenten lastet, immens, sind die Aufgaben, vor denen er steht, geradezu erdrückend. Gut möglich, dass wir dabei vielleicht gleich die nächste Krise im transatlantischen Verhältnis erleben, wenn allzu große Hoffnungen, wie sie mit dieser Wahl verbunden sind, am Ende enttäuscht werden. Einen radikalen Politikwechsel sollte man daher nicht zwangsläufig erwarten, auch nicht, dass dieser Präsident ein ,europäischer' sein wird. Der Grund hierfür liegt nicht nur in der politischen Agenda des neuen Präsidenten (die sich in vielerlei Hinsicht weit weniger stark von der des noch amtierenden abhebt), sondern auch darin, dass das Amerikabild schon vor dieser Wahl ein verzerrtes gewesen ist. Bereits die zweite Amtszeit von Bush hat mehr Gemeinsamkeiten mit Europa gebracht, als man diesseits des Atlantiks wahrhaben wollte. Dies gilt bezüglich der stereotypen Vorwürfe vom amerikanischen Unilateralismus und den fundamentalen außenpolitischen Differenzen im transatlantischen Verhältnis ebenso wie für die Wiederaufnahme des Dialogs mit Washington; immerhin besuchte Bush als erster amerikanischer Präsident die Europäische Kommission in Brüssel. ${ }^{1}$ Hinzu kommt, dass die Euro-

\footnotetext{
* Prof. Dr. Stefan Fröhlich, Professor für Internationale Politik, Friedrich-Alexander-Universität Erlangen-Nürnberg.

1 Rupert Cornwell: Bush Flies to Build Bridges with Europe's War Dissenters, in: The Independent on Sunday, 20.02.2008.
} 
päer schon längst nicht mehr nur reagierten, sondern zunehmend stärker in das internationale Krisenmanagement einbezogen wurden. Mit dem Iran verhandelt die EU-Troika Frankreich, Großbritannien und Deutschland auch im Namen der USA. Während der Georgien-Krise war es die französische Ratspräsidentschaft, die mit Russland eine Vereinbarung zur Beendigung des Krieges schloss, während Washington auffällig im Hintergrund blieb. Beim EUUS-Gipfel in Washington 2007 verständigten sich beide Seiten auf einen Prozess der Vertiefung des transatlantischen Wirtschaftsraumes. Und als die Finanzkrise ausbrach, war zunächst nicht nur die Zusammenarbeit zwischen den USA und Europa entscheidend, sondern auch die Europäische Union gewissermaßen der Schrittmacher, als auf beiden Seiten die finanziellen Rettungspakte geschnürt wurden; am Ende folgte Washington Großbritannien und anderen Mitgliedstaaten der Union, als man sich gar zu einer notwendigen Teilverstaatlichung der Kreditwirtschaft entschloss.

Dennoch blieb Washingtons Politik bei aller ,rhetorischen Konzilianz“ fixiert auf die Bekämpfung des internationalen Terrorismus, ,,militärische Überlegenheit und interventionistische Strategien".2 Auch deshalb geht es zunächst einmal um eine Veränderung in der Grundbefindlichkeit im bilateralen Verhältnis, wonach die generelle Ablehnung des außenpolitischen Kurses der USA der vergangenen Jahre zunehmend mit einem generellen Unbehagen bezüglich der inneren Verfasstheit Amerikas verbunden war. Die öffentliche Meinung in der Europäischen Union lehnt heute eine besondere amerikanische Führungsrolle mehrheitlich ab - und das, obwohl in der Beurteilung der Herausforderungen und Bedrohungen keine wesentlichen Unterschiede bestehen. ${ }^{3}$ Hoffnung besteht somit, dass ab 2009 wenn nicht alles anders, so doch vieles besser wird. Dies gilt nicht nur für Stil und Ton, auch mit Blick auf Substanz und die generelle Richtung wird erwartet, dass Washington wieder stärker auf seine Bündnispartner zugeht, multilateraler agiert und insgesamt mehr Zurückhaltung übt.

Umgekehrt aber wird dieses krisenbelastete Amerika, welches aus Kapazitäts- wie Legitimationsgründen noch lange brauchen wird, um nach Irak zu seiner alten Führungsstärke zu finden, unter dem neuen Präsidenten eine ,gerechtere ‘ Lastenteilung (höhere Verteidigungsausgaben) und mehr globales Engagement von den Europäern wünschen (Irak und Afghanistan), als diesen lieb sein dürfte - daran lassen die Äußerungen Obamas ebenfalls keinen Zweifel.

Wie wird die Agenda der neuen Administration aussehen und was bedeutet sie im Einzelnen für die Europäer? Zunächst vorab: Die Ära der uneingeschränkten amerikanischen Führung, der ,unipolare Moment"4 mag vorerst der Vergangenheit angehören und der Handlungsspielraum der USA stark eingeschränkt sein. Amerika wird sich aber unter Obama nicht aus der Weltpolitik verabschieden; auch zu seinem Selbstverständnis wird gehören, dass amerikanische Präsidenten zugleich die ,Führer der freien Welt' sind. Dies zeigte sich bereits in seinen Wahlkampfäußerungen, wonach die USA der Garant der internationalen Stabilität und die unentbehrliche Ordnungsmacht seien. Und nach wie vor gilt: Viele Probleme können die Amerikaner zwar nicht alleine lösen, aber kein Problem lässt sich eben ohne die amerikanische Unterstützung lösen. Trotz der gegenwärtigen Finanz- und Wirtschaftskrise haben die USA durch die einzigartige Kombination militärischer, wirtschaftlicher und technologischer Machtressourcen eine herausragende Position im globalen Sys-

2 Josef Janning: Welcome back, America? Die USA und Europa im Aufbruch, spotlight europe 12/2008.

3 Atlantic-community.org: Atlantic Faces. Jan Neutze, German Marshall Fund of the United States, 18.02.2008, abrufbar unter: http://www.atlantic-community.org/index/Global_Must_Read_Article/Jan_Neutze\%2C_Ger man_Marshall_Fund_of_the_United_States (letzter Zugriff: 18.12.2008).

4 Charles Krauthammer: The Unipolar Moment, in: Foreign Affairs 1/1991, S. 23-33. 
tem. Hinzu kommt, dass die Voraussetzungen für den künftigen Präsidenten gut sind, weil er nicht nur mit einer breiten Unterstützung für einen neuen Aufbruch in der Bevölkerung rechnen kann, sondern weil er auch über solide Mehrheiten im Kongress verfügt. Zwar erreichten die Demokraten mit 56 Sitzen $^{5}$ nicht ihr Ziel einer, filibuster-festen` Mehrheit von 60 der 100 Sitze im Senat, die die Möglichkeit der Minderheitenfraktion, mit ihrer Sperrminorität von zwei Fünfteln der Mandate ein Ende der Debatte und somit die Abstimmung über einen Gesetzentwurf zu verhindern, aufgehoben hätte. Immerhin aber scheinen die demokratischen Neuzugänge von ihrem Profil her diese Mehrheit in beiden Häusern nicht nach links zu verschieben, sondern eher in der politischen Mitte zu verankern. Eben dort lässt sich im Übrigen auch das Kabinett des 44. Präsidenten verorten.

\section{Neuer Pragmatismus und Entideologisierung amerikanischer Außenpolitik?}

Obama wird als erstes versuchen, die moralische Autorität Amerikas wiederherzustellen. Dazu gehören die sofortige Schließung des Gefangenenlagers von Guantanamo und damit verbunden die Wiederherstellung des ,Habeas-Corpus-Rechts` zur Anfechtung der Haftgründe vor einem zivilen amerikanischen Gericht, die Beendigung der bisherigen CIA-Praktiken wie die Entführung Terrorverdächtiger sowie die Ächtung jeder Form von Folter. Im Innern bedeutet dies eine grundlegende Reform des Gesundheitssystems, ein rigoroses Energiesparprogramm und eine Reform des Steuersystems, bei der die ,Superreichen ' zur Kasse gebeten und die Mittel- und Unterschicht entlastet werden, und vielleicht gar eine stärkere Regulierung und Disziplinierung der Wall Street. Bei allen Projekten bleibt der Präsident bislang die Antwort auf die Frage nach der Gegenfinanzierung schuldig, allerdings hat er signalisiert, dass ein weiterer Defizitanstieg in dieser Phase wohl unumgänglich sei.

Außenpolitisch bedeutet eine moralische Erneuerung, dass Obama sich eher verpflichtet fühlen wird, wieder verstärkt mit internationalen Organisationen und vor allem den europäischen Bündnispartnern zusammenzuarbeiten, das Völkerrecht nicht in der Weise wie sein Vorgänger zu missachten, sich vielleicht sogar auf verbindliche Richtlinien zur Begrenzung von Treibhausgasen einzulassen, dem Internationalen Strafgerichtshof beizutreten, auf eine Modernisierung des US-Nukleararsenals zu verzichten und entgegen seiner Ankündigung im Wahlkampf am Ende doch die Verteidigungsausgaben zu kürzen, um seine sozialpolitische Agenda durchsetzen zu können und die Wirtschaft zu stimulieren - und zwar aus purem amerikanischen Eigeninteresse heraus und nicht etwa aufgrund der Haltung der Europäischen Union. Ob es auch bedeutet, dass es tatsächlich zu dem zügigen Abzug aus dem Irak kommt, nur weil Obama die Vorstellungen der dortigen Bevölkerung respektiert; dass die USA auf ihre Sonderstellung bei der Erschließung der weltweiten Ölreserven verzichten; dass Washington eine Strategie für Afghanistan entwickelt, die eine neue Basis für den internationalen Terrorismus verhindert, aber neben militärischen Strafexpeditionen den zivilen Wiederaufbau viel stärker berücksichtigt und auch ungewöhnliche Schritte wagt wie die Einbeziehung, gemäßigter` Taliban in den Friedensprozess (immerhin konnte mit dieser Strategie auch der sunnitische Aufstand im Irak aus den Fängen von al-Qaida gelöst werden, was wesentlich zur Verbesserung der Sicherheitslage im Irak und zur entscheidenden Isolierung und Schwächung des Terrornetzes im Zweistromland beitrug, das seinen Kampf nun wieder auf seine Stammländer am Hindukusch zu konzentrieren scheint) - daran muss man

5 Zählt man auch den unabhängigen Demokraten, Senator Joseph Lieberman, und den unabhängigen Senator, Senator Bernard Sanders, zu den Demokraten (wie das häufig getan wird), verfügen die Demokraten über 58 Senatorenposten. 
zumindest auch Zweifel haben, wenn man die jüngsten Korrekturen Obamas an seinen bisherigen Positionen berücksichtigt.

Der Grund dafür ist einfach: Gerade in außenpolitischen Fragen entpuppt sich der neue Präsident als Pragmatiker und beinahe schon postideologische Figur; ein Blick in sein Buch „The Audacity of Hope“ zeigt, dass es darin weniger um die Themen ,Hoffnung und Wan-

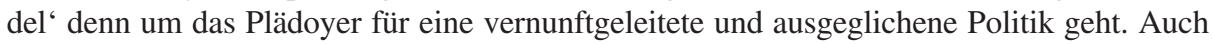
Obama steht durchaus für die Vision universaler Freiheit. Bei deren Umsetzung setzt er jedoch weniger auf die unter Bush forcierte Institutionalisierung von Demokratien im Sinne westlicher Ordnungsvorstellungen durch ,regime building', sondern auf die Überzeugung von einem liberalen und pluralen Amerika, das die Ideale aller Weltreligionen und Zivilisationen vereint: nämlich Gerechtigkeit, Freiheit und ein guter Lebenswandel, nicht zuletzt im Sinne eines friedfertigen Umgangs miteinander. ${ }^{6}$ Anders als sein Vorgänger spaltet er die Welt nicht in ,Gut und Böse', sondern präsentiert sich als Mann des internationalen Ausgleichs, der sozialen Balance und der Integration. Gegen den Irakkrieg wandte er sich nicht mit dem Standardargument links-liberaler Kritiker von der generellen Ablehnung von Kriegen. Vielmehr kritisierte er das überstürzte Handeln der Regierung, eine Politik, die von „Emotionen und nicht von Prinzipien“ geleitet war und die mittel- bis langfristigen Konsequenzen für das Ansehen Amerikas in der Welt ignorierte. ${ }^{7}$

Zwar hält er an der Grundüberzeugung fest, wonach Amerika aufgrund seiner überragenden Machtposition die internationale Ordnung gestalten kann und wonach die Gestaltung der inneren wie äußeren Ordnung im Sinne von Karl W. Deutsch zentrale Aufgabe aller Staatlichkeit ist. ${ }^{8}$ Und da dieser Gestaltungswille wesentlich von den religiösen Fundamenten und Werten (Freiheit, Demokratie, Menschenrechte, Toleranz, Respekt, Solidarität, Ziel- und Ergebnisorientiertheit) seiner Gesellschaft mitgetragen wird, welche wiederum als quasi natürlicher Wunsch aller Zivilgesellschaften vorausgesetzt werden, wird auch Obama sich mit Nachdruck für deren Bewahrung und Strahlkraft einsetzen. Dabei wird er diesen Einsatz jedoch nicht zu einem generellen normativen Konflikt zwischen dem demokratischen Amerika auf der einen und undemokratischen Regimen, die die Sicherheit des Landes bedrohen, auf der anderen Seite hochstilisieren. Der Aufstieg des transnationalen radikalen Islamismus hat zwar nach Ansicht Obamas das Paradigma säkularer internationaler Beziehungen erschüttert und stellt eine zentrale Herausforderung für den Westen insgesamt dar. Ebenso sieht er, dass der Universalitätsanspruch liberaler Demokratie durch die Betonung fremder kultureller Eigenheiten und vor allem Russlands und Chinas Autoritarismus zunehmend infrage gestellt wird und Washington sich daher auch aus diesem Grund von der Idee der Erzwingung westlicher Ordnungsmodelle verabschieden muss.

Andererseits räumt er neben der Bedrohung durch den islamistischen Terrorismus und die Verbreitung von Massenvernichtungswaffen den Herausforderungen durch schwache und scheiternde Staaten (als Ursache von Instabilität), extreme Armut, Klimawandel und Energieknappheit den gleichen Stellenwert ein und sieht deshalb die amerikanische Sicherheit und den Wohlstand untrennbar mit der Sicherheit und dem Wohlergehen in anderen Staaten verbunden. ${ }^{9}$ Aus dieser Verbundenheit heraus ergibt sich für ihn die Notwendigkeit

6 Barack Obama: The Audacity of Hope. Thoughts on Reclaiming the American Dream, New York 2006, S. 316-317.

7 Barack Obama: Text of Senator Barack Obama's Speech at Anti-War Rally in Chicago, 02.10.2002, abrufbar unter: http://www.barackobama.com/pdf/ObamaIraqHandout.pdf (letzter Zugriff: 18.12.2008).

8 Karl W. Deutsch: Politische Kybernetik. Modelle und Perspektiven, Freiburg 1970.

9 Peter Rudolf: Amerikas neuer globaler Führungsanspruch. Außenpolitik unter Barack Obama, SWP-Aktuell $77 / 2008$. 
der Partnerschaft und der Führung durch das eigene Beispiel im Sinne der Jeffersonschen Tradition in der amerikanischen Außenpolitik.

Mit anderen Worten: Obama wird sich der Welt weit weniger moralistisch und idealistisch präsentieren als sein Vorgänger und auch John McCain. In den vergangenen Monaten hat er wiederholt betont, dass er einen ,,außenpolitischen Realismus“ jeder ,,ideologisierten Außenpolitik“ vorziehe. Dabei bediente er sich außenpolitischer, Champions" der Demokraten wie Franklin D. Roosevelt, Harry S. Truman, Dean Acheson oder John F. Kennedy ebenso wie der republikanischen ,Garde' um George F. Kennan, Brent Scowcroft, James Baker oder auch George H.W. Bush. Beobachter charakterisierten ihn bereits während des Wahlkampfes als den „,cool conservative“ neben dem ,exuberant idealist“ McCain. ${ }^{10}$ Doch auch Obama steht in einer außenpolitischen Tradition, in der Idealismus und Realismus, Moral und Macht beziehungsweise Interessen miteinander verschmelzen. Er will die USA daher zurückführen in die Rolle des liberalen und ,wohlwollenden Hegemons', wie sie den Entwurf amerikanischer Weltpolitik nach 1945 prägte. Danach können die USA aufgrund ihrer Ressourcen zwar die eigenen Interessen unilateral verfolgen, sind sich aber andererseits ihrer besonderen globalen Verantwortung für die Stabilität des internationalen Systems bewusst und beschränken daher den Einsatz ihrer militärischen Macht nicht auf den Schutz der amerikanischen Bevölkerung und vitaler Interessen in Fällen tatsächlich oder unmittelbar bevorstehender Angriffe. Dementsprechend will Obama bis 2012 die amerikanische Auslandshilfe pro Jahr auf 50 Milliarden US-Dollar verdoppeln, gleichzeitig aber auch die Verteidigungsausgaben und die Personalstärke der Streitkräfte (um 90.000) erhöhen - nicht zuletzt zur effektiveren Bekämpfung von Aufständen beziehungsweise Umsturzversuchen in „schwachen“, ,scheiternden“ oder „gescheiterten“ Staaten sowie zur Unterstützung beziehungsweise zum Wiederaufbau von Streitkräften in den betroffenen Ländern (,post-conflict-management“) ${ }^{11}$ Insofern könnte die Option des Einsatzes militärischer Macht auch im Falle humanitärer Katastrophen und im Dienste ,gemeinsamer Sicherheit“, möglichst im Rahmen internationaler Institutionen, aber auch mit Unterstützung anderer Staaten in flexiblen „Koalitionen von Handlungswilligen“, wenn der Sicherheitsrat der Vereinten Nationen (VN) sich als handlungsunfähig erweist, zum Eckpunkt einer künftigen Obama-Doktrin werden. ${ }^{12}$ Nicht zuletzt Äußerungen aus dem engeren Beraterumfeld des künftigen Präsidenten, so etwa von der designierten US-Botschafterin bei den Vereinten Nationen, Susan E. Rice, oder von Anthony Lake, deuten darauf hin, dass das Zeitalter des Unilateralismus mit dem Ende der Ära Bush keinesfalls endgültig aus dem außenpolitischen Instrumentenkasten der USA verschwunden sein wird. ${ }^{13}$

\section{Obamas neuer Multilateralismus}

Amerika wird unter Obama somit nicht vor globaler Verantwortung zurückweichen oder gar isolationistischer werden. Die Grundprinzipien beziehungsweise Parameter amerikanischer Außenpolitik bleiben bestehen: die innere Sicherheit bleibt ein zentrales Thema; Obama hält die Bedrohung durch Massenvernichtungswaffen und internationalen Terrorismus unverändert für die größte unmittelbare sicherheitspolitische Herausforderung des Lan-

10 Fared Zakaria: Obama Abroad, in: Newsweek, 28.07.2008.

11 Barack Obama: Defense, abrufbar unter: http://www.barackobama.com/issues/defense/ (letzter Zugriff: 18.12.2008); Barack Obama: Renewing American Leadership, in: Foreign Affairs 4/2007, S. 2-16, hier S. 7.

12 Michael Fullilove: Hope or Glory? The Presidential Election and U.S. Foreign Policy, Brookings Foreign Policy Paper 9/2008, S. 6-7.

13 Susan E. Rice/Anthony Lake/Donald M. Payne: We saved Europeans. Why Not Africans?, in: The Washington Post, 02.10.2006; Barack Obama/Hillary Clinton/John McCain: We Stand United on Sudan, 28.05.2008, abrufbar unter: http://www.savedarfur.org/page/content/Candidates_Statement/ (letzter Zugriff: 18.12.2008). 
des (und der westlichen Welt); Irak und Afghanistan stehen unverändert an vorderster Stelle der außenpolitischen Agenda; auch Obama hält unilaterales Handeln und Präemptivschläge in bestimmten, die nationale Sicherheit Amerikas bedrohenden Situationen für notwendig, sollte die internationale Staatengemeinschaft zu geschlossenem Handeln nicht in der Lage sein; ${ }^{14}$ auch er sieht in der bestmöglichen Ausstattung der US-Streitkräfte die Voraussetzung für die Garantie der Sicherheit Amerikas und seiner Verbündeten. Zu einer Abkehr vom bisherigen Kurs scheint er hingegen vor allem in Bezug auf die amerikanische Energiepolitik (binnen der nächsten zehn Jahre will er das Land von ausländischen Energielieferungen unabhängig machen, indem er unter anderem auch die umstrittene Ölförderung im eigenen Land fördern will, aber auch auf erneuerbare Energien setzt) sowie die Klimapolitik bereit zu sein.

Die größten Hoffnungen der Europäer ruhen daher auf der Einsicht in Washington, dass die USA nicht zuletzt aus Gründen ihres ramponierten Images in der Welt, aber auch aus Legitimationszwängen gegenüber der eigenen Bevölkerung heraus und wegen fehlender eigener Mittel wieder mehr die Unterstützung und Zusammenarbeit mit den VN suchen werden. Obama hat deutlich gemacht, dass sein Vertrauen in die Möglichkeiten der VN begrenzt ist, dass Washington aber die eigenen Ziele in den meisten Fällen mit deren Unterstützung leichter durchsetzen kann und als globale Ordnungsmacht mehr als andere Nationen von der Beachtung ihrer Regeln und Werteordnung profitiert. ${ }^{15} \mathrm{Zu}$ diesen Zielen gehören für Obama zunächst ganz allgemein die Anpassung der von den USA geschaffenen multilateralen Strukturen und Institutionen an ein verändertes internationales Umfeld, sprich die Durchsetzung institutioneller Reformen, und die stärkere Einbindung von nichtstaatlichen Akteuren. In diesem Zusammenhang sprach Obama bereits während des Wahlkampfes gerne von der „globalisierten Welt“ und „transnationalen Bedrohungen“, welche nach ,integrativen und kooperativen Lösungen“" verlangten (,,interconnectivity“). ${ }^{16}$

Dazu zählt auch die stärkere Integration anderer Staaten in multilaterale Institutionen, so insbesondere Chinas, Indiens oder Brasiliens, aber eben auch der europäischen Bündnispartner, nicht zuletzt um einen Teil der mit der amerikanischen Führungsrolle verbundenen Kosten auf diese abwälzen zu können. Nichts anderes bedeutete die während des Wahlkampfes immer wieder erhobene Forderung an die Europäer zu mehr Anstrengungen für die globale Sicherheit.

In diesem Zusammenhang signalisiert Washington auch die Bereitschaft zur Einrichtung neuer Institutionen; vor allem mit Blick auf den Aufstieg Chinas wird intensiv über die Möglichkeit neuer multilateraler Sicherheitsstrukturen für die asiatisch-pazifische Region nachgedacht, wohlgemerkt in der Erwartung chinesischer Bereitschaft, die dortige sicherheitspolitische Führungsrolle der USA als Garant für Stabilität anzuerkennen. Obama hat angekündigt, in Anknüpfung an die bestehenden bilateralen Bündnisse und die ,Sechs-Parteien-Gespräche ‘ zu Nordkorea einen „effektiven regionalen Rahmen“ in Asien zu schaffen, nicht zuletzt, um den sicherheitspolitischen Bedenken seiner Verbündeten Südkorea, Philippinen, Thailand und Indonesien hinsichtlich einer möglichen militärischen Bedrohung durch China zu begegnen. Gleichwohl signalisieren seine Äußerungen bezüglich der Menschenrechtssituation im Lande ansonsten, dass Obama vor allem ein sehr pragmatisches Interesse an der Aufrechterhaltung möglichst reibungsloser ökonomischer Beziehungen zu Peking hat. ${ }^{17}$

14 Obama: The Audacity of Hope, 2006, S. 308-309.

15 Ebenda.

16 Barack Obama: Remarks of Senator Barack Obama: A World that Stands as One, 24.07.2008, abrufbar unter: http://www.barackobama.com/2008/07/24/remarks_of_senator_barack_obam_97.php (letzter Zugriff: 18.12.2008).

17 Barack Obama: Statement on the Occasion of the Visit of Vice Premier Wu Yi, in: Congressional Records Senate, 23.05.2007, S. S6545-S6546. 
Ähnlich pragmatisch verfährt er auch mit Blick auf Russland. Weder verschließt er die Augen vor der demokratischen Erosion im Lande, noch riskiert er deshalb seine Strategie einer selektiven Kooperation mit Moskau. Anerkennung russischer Statusansprüche bei gleichzeitigem Festhalten an den Zielen der NATO-Erweiterung und gegebenenfalls der Raketenabwehrpläne, so lautet das Motto. Aus diesem Grund lehnte Obama bereits McCains Vorschlag eines Ausschlusses Russlands aus der G8 und der Welthandelsorganisation ab und hielt sich zunächst auch während der Georgien-Krise im August 2008 mit allzu offener Kritik gegenüber Moskau zurück. ${ }^{18}$ Einer der Gründe: Obama hat sich das ehrgeizige Ziel gesetzt, die Führungsrolle der USA in der nuklearen Abrüstungs- und Nichtverbreitungspolitik zu erneuern und dabei gar die Vision einer vollständigen nuklearen Abrüstung aufzugreifen - jene nie ernst genommene Perspektive des Nichtverbreitungsvertrags, für die sich bereits Anfang 2007 eine Gruppe prominenter Außen- und Sicherheitspolitiker um Henry A. Kissinger und George P. Shultz herum einsetzte. ${ }^{19}$ Will er dabei nur annähernd Erfolg haben und mit seiner Politik der wachsenden Bedrohung durch Nuklearwaffen im Besitz einer größeren Zahl von Staaten und vor allem von Terroristen vorbeugen, braucht er in jedem Fall Moskaus Unterstützung - zumal, wenn er Kritikern im eigenen Land begegnen will, die keine Hoffnung hegen, dass Veränderungen in der amerikanischen Nuklearstrategie Staaten wie Iran, Nordkorea, Indien oder Pakistan zur Aufgabe ihrer eigenen Nuklearprogramme bewegen könnten.

Obamas Pragmatismus geht aber eben auch nicht so weit, dass er deshalb die einseitige nukleare Abrüstung zum Ziel hat. An einer starken nuklearen Abschreckung hält er fest, solange es Atomwaffen gibt. Erst vor wenigen Wochen bestätigte der US-Kongress diese Einschätzung in einer Studie zur aktuellen wie künftigen Terrorgefahr, indem er vor der wachsenden Gefahr von „Schurkenstaaten“, Atomschmugglern und der weltweiten Verbreitung der Atomtechnologie warnte. Dabei zeigten sich die Autoren besonders besorgt über die Atommacht Pakistan, die man als große Gefahr und ,unwissentliche Quelle“ eines Terrorangriffs auf die USA bezeichnet. ${ }^{20}$ Dennoch oder gerade deshalb will Obama die aus dem Nichtverbreitungsvertrag resultierende Abrüstungsverpflichtung für die Nuklearstaaten wieder ernster nehmen als seine Vorgänger. Sowohl Bill Clinton wie auch Bush hielten bei ihren Überprüfungen der Nuklearpolitik jeweils an der Grundmaxime fest, wonach eine große Zahl von Atomwaffen als Vorsichtsmaßnahme gegenüber Russland und auch China notwendig ist. Während Clinton darüber hinaus die US-Atomwaffen zusätzlich in den Dienst der Abschreckung eines möglichen Angriffs durch biologische und chemische Waffen stellte, kam es unter Bush auch zur Entwicklung neuer nuklearer Sprengköpfe zur Ausschaltung unterirdisch gelegener Ziele. Obama aber drängt auf eine „dramatische Reduzierung“ der nuklearen Arsenale, auf eine globale Ausweitung des Mittelstreckenvertrags und ein globales Verbot der Produktion waffenfähigen spaltbaren Materials. Aus diesem Grund will er einen Strategiewechsel, wonach die amerikanischen Nuklearwaffen künftig ausschließlich dem Zweck der Abschreckung des Einsatzes selbiger durch andere Staaten dienen. Auf diese Weise könnte man eine Reduzierung der Zahl der atomaren Gefechtsköpfe von derzeit

18 Barack Obama: Statement on Russian Presidential Elections, 03.03.2008, abrufbar unter: http://www.barackobama.com/2008/03/03/statement_of_senator_barack_ob_6.php (letzter Zugriff: 18.12.2008); Barack Obama: Statement of Senator Barack Obama on the Conflict in Georgia, 11.08.2008, abrufbar unter: http://www.barackobama.com/2008/08/11/statement_of_senator_barack_ob_22.php (letzter Zugriff: 18.12.2008).

19 George P. Shultz/William J. Perry/Henry A. Kissinger/Sam Nunn: Toward a Nuclear-Free World, in: The Wall Street Journal, 15.01.2008; George P. Shultz/William J. Perry/Henry A. Kissinger/Sam Nunn: A World Free of Nuclear Weapons, in: The Wall Street Journal, 04.01.2007.

20 Joby Warrick: Nuclear or Biological Attack Called Likely, in: The Washington Post, 02.12.2008. 
7.000 auf etwa 1.000 erreichen und damit auch einen wichtigen Schritt in Richtung eines glaubwürdigen und vor allem überschaubaren nuklearen Kontrollregimes.

Ebenso wenig naiv ist Obama mit Blick auf neuerliche Moskauer Versuche, mit dem russisch-georgischen Krieg im Südkaukasus auf die alten Gleise sowjetischer Machtpolitik zurückzukehren, Antiamerikanismus zur Legitimierung innenpolitischer Macht zu betreiben, russische Energieträger als politische Instrumente einzusetzen oder im ,Nahen Ausland “ eigene Interessen mit großer Härte durchzusetzen. Obama hat signalisiert, dass Dmitrij Medwedjews Ankündigung, Moskau werde in Kaliningrad Kurzstreckenraketen aufstellen sollte Bushs Nachfolger an dessen Plan festhalten, in Polen und in der Tschechischen Republik ein Raketenabwehrsystem zu installieren -, sehr rasch zu einer außenpolitischen Isolierung Russlands vom Westen und einer Vereisung der Beziehungen zu den USA führen könne; bestärkt wurde er dabei auch von der eher ablehnenden Haltung der GUS-Staaten, wie etwa Armenien oder Aserbaidschan, gegenüber Moskaus Vorstoß im Kaukasus. Das von Medwedjew präsentierte Konzept einer ,neuen Sicherheitsarchitektur' (gleiche Sicherheit für alle, Regelung des Einsatzes von Gewalt, Rüstungskontrolle und Bezug zur VNCharta), mit dem Moskau über ein russisches Vetorecht seinen Einfluss in Europa zu vergröBern sucht, dient auch aus Sicht des künftigen US-Präsidenten vor allem dem Zweck, einen Keil zwischen Europa und Amerika zu treiben, um so den Fortgang der NATO-Erweiterung und eben die Stationierung des amerikanischen Abwehrsystems auf mitteleuropäischem Boden zu verhindern. Nicht anders sind Medwedjews Worte zu verstehen, wonach der alte „Atlantizismus“ am Ende sei und nunmehr ein „neuer euro-atlantischer Ansatz“ als Ausdruck der neuen multipolaren Welt verfolgt werden müsse. ${ }^{21}$

Entscheidend sind zwei Fragen: Erstens, wie weit reicht Obamas so verstandener pragmatischer Multilateralismus? Und zweitens, welche Möglichkeiten für eine gemeinsame, zumindest koordinierte Russland-Politik Amerikas und der Europäischen Union gibt es vor dem Hintergrund der gleichsam unterschiedlichen Positionen beider Seiten in Bezug sowohl auf die NATO-Erweiterung wie auch die Raketenabwehrpläne? Im ersten Fall hieße das nichts anderes, als beispielsweise ein Handel mit Moskau nach dem Motto ,Verzicht auf beide Pläne gegen robustes russisches Einwirken etwa auf den Iran'. Im zweiten Fall bedeutete es, dass sich Washington unter dem neuen Präsidenten von der Position seines Vorgängers distanziert, wonach eine Entscheidung über die Aufnahme der Ukraine und Georgiens in das Vorbereitungsprogramm der NATO für eine spätere Mitgliedschaft nunmehr unmittelbar anstünde.

Auch in anderen Fragen bedeutet Amerikas neuer Multilateralismus keinesfalls eine Annäherung an ein europäisches Verständnis davon. Das amerikanische Verständnis von Multilateralismus wird auch unter Obama zunächst ein instrumentelles sein. Dort, wo amerikanische Interessen unmittelbar tangiert sind, wird Washington im Einzelfall abwägen, ob es diese globalen unterordnet. Deutlich wurde dies bereits mit der vorsichtigen Position zum Internationalen Strafgerichtshof, in der sich vor allem die (sicherheits-)politischen Bedenken der US-Militärs spiegeln. Zur Unterzeichnung des Rom-Statuts wollte Obama sich vor Amtsantritt jedenfalls nicht äußern.

Viel problematischer könnte Obamas Multilateralismus-Verständnis jedoch ausgerechnet im Verhältnis zu den europäischen Bündnispartnern werden. Umfragen mögen zwar signalisieren, dass Obama in den Augen der Europäer nicht nur als großer Kommunikator, sondern

21 Dmitrij Medwedjew: Address to the Federal Assembly of the Russian Federation, 05.11.2008, abrufbar unter: http://www.kremlin.ru/eng/speeches/2008/11/05/2144_type70029type82917type127286_208836.shtml (letzter Zugriff: 18.12.2008). Vgl. auch Frankfurter Allgemeine Zeitung, 06.11.2008. 
auch als ein quasi kongenialer Präsident betrachtet wird. ${ }^{22}$ Dabei wird jedoch zweierlei übersehen: Erstens ist keinesfalls klar, dass Europa bei der Frage nach möglichen amerikanischen Kooperationspartnern und Verbündeten immer die erste Wahl für den Präsidenten sein wird; bereits während des Wahlkampfes - in logischer Konsequenz seines politischen Pragmatismus - hat er wiederholt zu erkennen gegeben, dass er Allianzen weniger in engen beziehungsweise vertrauten Kategorien denkt, sondern eher instrumentell sieht, also im Sinne des Mittels effektiven globalen Regierens. So sprach er zwar offen von der Absicht der Wiederbelebung von Allianzen, gleichzeitig aber auch über die Neueinrichtung von „Partnerschaften und Institutionen“. ${ }^{23}$ Zweitens sieht er darin auch ein Mittel des , burdensharings', das heißt Kosten auf andere Staaten abzuwälzen. Damit ist die bereits viel zitierte Frage nach einer möglichen stärkeren Einbindung der Europäer angesprochen, so etwa bei der Unterstützung im Irak, in Afghanistan oder im Iran. Partnerschaft und ,geteilte" Verantwortung im Dienste eines sicheren Amerikas und des Westens insgesamt, so lautet die Losung; im Gegenzug darf Europa wieder mit mehr Respekt seitens des Bündnispartners in Washington rechnen.

Wie diese Unterstützung beziehungsweise neue Lastenteilung im Einzelnen aussehen soll, insbesondere zur Sicherung der Stabilität im erweiterten Krisenbogen vom Maghreb bis in den Persischen Golf und darüber hinaus in die zentralasiatische Region, sprich des sogenannten ,Greater Middle East', ist bislang unklar. Fest steht aber, dass sie sehr rasch ein Thema in den transatlantischen Beziehungen wird, bei dem die Europäer ihrerseits mit klaren Vorstellungen in Verhandlungen mit Washington hineingehen sollten. Amerika wird seine überragende Militärpräsenz zur Sicherung der freien Ölzufuhr und stabiler Verhältnisse in der Region sicherlich nicht aufgeben; es wird aber dabei versuchen, sich erstens der Region mit seinen ordnungspolitischen Vorstellungen nicht weiter als nötig aufzudrängen, und es wird außerdem alles unternehmen, um die sich daraus ergebenden enormen finanziellen Belastungen für Washington zu senken. Das Instrument für diese Strategie sieht man in der Schaffung einer regionalen kollektiven Sicherheitsarchitektur - ein Vorschlag, mit dem die Europäische Union bereits wiederholt in Washington vorstellig geworden ist $-{ }^{24}$ in der neben den Staaten der Region und den Europäern auch China, Indien und eventuell Russland einen Teil der Kosten übernehmen und wenn möglich auch militärisch präsent sein sollen. Mit anderen Worten, ,Entamerikanisierung', bei gleichzeitiger Regionalisierung lauten die Mittel, mit denen Washington seine Militärpräsenz sukzessive auf ein Mindestmaß reduzieren will. Dabei sollen alle Staaten der Region, Syrien und Iran inbegriffen, einbezogen werden.

\section{Außenpolitische Herausforderungen und Möglichkeiten transatlantischer Annähe- rungen}

Irak und der Umgang mit anderen , unfreundlichen Regimen` wie Iran, Syrien oder Nordkorea sind die Themen, bei denen sich Obama nicht nur von seinem Kontrahenten unterschied, sondern bei denen durchaus auch Potenzial für eine verbesserte transatlantische Zusammenarbeit besteht. Dies gilt hingegen nur bedingt für die Themen Freihandel und Klimawandel - obwohl sich gerade mit letzterem vielleicht die größten europäischen Hoff-

22 GlobeScan Incorporated/The Program on International Policy Attitudes (PIPA)/The BBC: All Countries in BBC Poll Prefer Obama to McCain, BBC World Service Poll, 10.09.2008, abrufbar unter: http:// news.bbc.co.uk/2/shared/bsp/hi/pdfs/10_09__08_ws_us_poll.pdf (letzter Zugriff: 18.12.2008).

23 Obama: Renewing American Leadership, 2007, S. 11; John R. Bolton: One world? Obama's on a different planet, in: Los Angeles Times, 26.07.2008.

24 Vgl. Stefan Fröhlich: Die EU als globaler Akteur. Eine Einführung, Wiesbaden 2008, S. 250-269. 
nungen verbinden. Im Falle Iraks braucht Obama sich kaum noch an seiner Absicht messen zu lassen, den Truppenrückzug aus dem Irak binnen 16 Monaten nach Amtsübernahme vollendet zu haben; ein entsprechendes Abkommen ist nach der Annahme des Abkommens über den Rückzug der amerikanischen Truppen bis 2011 durch das irakische Parlament mittlerweile noch unter seinem Vorgänger ausgehandelt worden. Das heißt aber umso mehr, dass Amerika sich fortan stärker der inneren Verfasstheit des Landes widmen wird. Obama hat signalisiert, dass er den Irak weiter unterstützen will, eine fundamental andere Orientierung zu entwickeln. Gleichzeitig setzt er auf einen nachhaltigen Sinneswandel in den USA, der künftigen Interventionen wie im Zweistromland vorbeugt; in verschiedenen Reden hat er dabei seine Überzeugung deutlich gemacht, dass das Land Krisen immer dann hervorgerufen habe, wenn es seine militärische Macht am falschen Ort und zum falschen Zeitpunkt einsetzte, nicht hingegen, wenn man auf diesen Einsatz verzichtete. ${ }^{25}$ Sollte sich wider Erwarten die Lage im Irak jedoch wieder verschlechtern, so stehen die USA auch unter dem neuen Präsidenten bereit. Darauf deutete seine noch vor den Wahlen gemachte Äußerung hin, Abzugspläne je nach den Gegebenheiten vor Ort und der Absprache mit den US-Militärs und der irakischen Regierung richten zu wollen.

Fest hält Obama unverändert an dem Vorschlag Joe Bidens, den Irak in drei faktisch unabhängige Gliedstaaten - einen kurdischen im Norden, einen sunnitischen in der Mitte und einen schiitischen im Süden - zu teilen. Der Vorschlag ähnelt der vom damaligen Balkan-Unterhändler Clintons, Richard C. Holbrooke, ausgearbeiteten Daytoner Friedenslösung für Bosnien und Herzegowina von 1995, die Biden seinerzeit im Kongress maßgeblich verteidigt hatte. 13 Jahre nach diesem Vertrag ist das Land mit seinen faktisch unabhängigen, wesentlich ethnisch definierten ,Entitäten " und einer schwachen Zentralregierung allerdings kaum ein Modell für die Befriedung eines ethnisch, religiös und auch sprachlich so vielfältigen Staates wie der Irak.

Im Falle Afghanistans fordert Obama zusätzlich 7.000 amerikanische Soldaten - darunter etwa jene, die aus dem Irak abgezogen wurden - sowie weitere Truppen der NATO-Verbündeten im Lande, um so den ,richtigen Krieg“ gegen die Taliban und al-Qaida am ,richtigen Ort" führen zu können. ${ }^{26}$ Das sind zwar weniger als die 20.000, die Bush mittlerweile vorsieht, zeigt aber, dass er es mit der Prioritätenverschiebung ernst meint. Der Umstand, dass seit Monaten mehr amerikanische Soldaten in Afghanistan fallen als im Irak, verleiht seiner Forderung zusätzliche Dringlichkeit. Allerdings wird die Aufstockung mit einem stärker regionalen Ansatz kombiniert, bei dem zum einen die afghanischen Sicherheitskräfte (zusätzliche Kosten 17 Milliarden US-Dollar) gestärkt, zum anderen, wie von Kabul angeregt, Teile der Taliban in den Dialog einbezogen werden sollen. Der Krieg soll somit zumindest teilweise ,entamerikanisiert", vor allem aber regionalisiert werden - ein Ansatz, der durchaus europäischen Vorstellungen entspricht. Ob mit dieser Neuausrichtung der Strategie in Afghanistan, maßgeblich vom neuen Chef des Zentralkommandos der US-Streitkräfte, General David Petraeus, entwickelt, auch die Forderung vom Tisch ist, die NATO-Bündnispartner sollten sich stärker in Afghanistan engagieren und ihre operationellen und räumlichen Beschränkungen (,caveats') aufgeben, bleibt abzuwarten. ${ }^{27}$ Die Europäer müssen

25 Barack Obama: Remarks of Senator Barack Obama: A New Strategy for a New World, 15.07.2008, abrufbar unter: http://www.barckobama.com/2008//07/15/remarks_of_senator_barack_obam_96.php (letzter Zugriff: 18.12.2008); Barack Obama: My Plan for Iraq, in: The New York Times, 14.07.2008.

26 Time Magazine Online: McCain and Obama on Afghanistan, 17.07.2008, abrufbar unter: http:// www.time.com/time/magazine/article/0,9171,1823945,00.html (letzter Zugriff: 18.12.2008).

27 SpiegelOnline: Obama Calls for Help from NATO Allies in Afghanistan, 29.02.2008, abrufbar unter: http:// www.spiegel.de/international/world/0,1518,538578,00.html (letzter Zugriff: 18.12.2008); Andrew Ward: Obama pledges to focus on Afghanistan, in: Financial Times, 20.07.2008. 
sich jedenfalls auf Forderungen nach deutlicheren Beiträgen einrichten; absehbar ist jedoch, dass die alten Grundmuster in der transatlantischen Lastendebatte sehr rasch wieder auftauchen, wonach die Europäer auch gegenüber Obama vorsichtig darauf hinweisen werden, dass Afghanistan nicht mit militärischen Mitteln zu gewinnen ist, und deshalb nur das in ihren Augen ,Nötigste` zur Entlastung Washingtons tun werden, während die USA über die schwachen und säumigen Europäer räsonieren.

Dass ,Entamerikanisierung ' nicht gleich Rückzug bedeutet, darauf weisen auch Obamas Pläne bezüglich der weiteren Entwicklungen in Pakistan hin. Man darf gespannt sein, wie sich seine Unterstützung von gezielten Operationen amerikanischer Soldaten auf pakistanischem (und syrischem) Boden, wie sie bereits seit 2004 durch US-Geheimkommandos im Anti-Terrorkampf durchgeführt werden, eben mit dieser Strategie der Einbindung gemäßigter Taliban in den Befriedungsprozess vereinbaren lässt. Obama hat solche Angriffe als ,genau das“ bezeichnet, was er mit dem Satz meinte, er wolle, ,,wenn wir Informationen über den Aufenthaltsort von Osama bin Laden oder anderer al-Qaida-Mitglieder haben, und die pakistanische Regierung ist unwillig oder unfähig zu handeln“, dann eben selbst handeln. Er ist der Meinung, dass der Krieg in Afghanistan nicht gewonnen werden kann, ohne das neu entstandene Lagernetzwerk im äußersten Nordwesten Pakistans zu zerschlagen; dafür will er Pakistan militärisch und wirtschaftlich helfen. Islamabad allerdings hat bereits signalisiert, dass es solche Operationen ablehnt und dementiert den Hinweis Washingtons, wonach es eine entsprechende Vereinbarung zwischen beiden Seiten bezüglich solcher Übergriffe gäbe.

Ähnliche Entwicklungen gibt es vor allem in Bezug auf Syrien und, wie einige Washingtoner Beobachter mutmaßen, gar mit Blick auf Iran (zum Ausspähen etwaiger militärischer Ziele für einen Schlag gegen die Atomanlagen des Landes). Auch dort kam es in den vergangenen Wochen zu Einsätzen amerikanischer Spezialeinheiten gegen mutmaßliche Islamistenlager, die allerdings von Damaskus nicht grundsätzlich kritisiert wurden, da sie sich gegen Staatsfeinde, nicht gegen staatliche Kräfte richteten. Die von Obama geforderten direkten Gespräche mit Syrien und auch mit dem Regime in Teheran sind für die künftige Administration auf jeden Fall eine ernsthafte Option und die Europäische Union muss sich positionieren, ob sie im Falle weiterer iranischer Verweigerung zu konstruktiven Gesprächen diesen Neuansatz unterstützt. Allerdings betont auch Obama inzwischen, dass er sich nicht zu Propagandazwecken missbrauchen lassen würde und dass seine Bereitschaft zu Gesprächen mit Syrien und Iran, oder auch mit Kuba, Venezuela und Nordkorea nicht nur von vorherigen gründlichen Sondierungen, sondern auch davon, dass er über Ort und Zeitpunkt solcher Gespräche bestimmt, abhinge. ${ }^{28}$ Eine Nuklearmacht Iran ist für ihn auf keinen Fall hinnehmbar und muss notfalls mit allen Mitteln verhindert werden; eine Verschärfung der Sanktionen auch außerhalb der VN ist somit das Mindeste, was auch er fordert. ${ }^{29}$ Gleichzeitig schließt er militärische Operationen nicht aus, hat aber bereits im Wahlkampf signalisiert, dass er solche derzeit für einen ,großen Fehler“ hielte. In diesem Kontext setzt er sich vorbehaltlos für das Existenzrecht Israels ein, lehnt Verhandlungen mit der Hamas bislang ab und plädiert für eine Wiederbelebung des Nahostfriedensprozesses. ${ }^{30}$

28 Jim Ruttenberg/Jeff Zeleny: Obama Seeks to Clarify His Disputed Comments on Diplomacy, in: The New York Times, 29.05.2008.

29 Barack Obama: Statement of Senator Barack Obama on Additional Sanctions on Iran, 04.03.2008, abrufbar unter: http://www.barackobama.com/2008/03/04/statement_of_senator_barack_ob_7.php (letzter Zugriff: 18.12.2008).

30 Barack Obama: Remarks of Senator Barack Obama: AIPAC Policy Conference, 04.06.2008, abrufbar unter: http://www.barackobama.com/2008/06/04/remarks_of_senator_barack_obam_74.php (letzter Zugriff: 18.12.2008) 
Neben den großen sicherheitspolitischen Herausforderungen bleiben zwei weitere Punkte, die im transatlantischen Verhältnis unter dem neuen Präsidenten für viel Potenzial, aber auch Konfliktstoff stehen könnten: die Außenwirtschafts- beziehungsweise Handelspolitik und die Klimapolitik. Obama folgt bislang eher der protektionistischen Grundhaltung in der Demokratischen Partei und im Kongress. NAFTA ${ }^{31}$ soll unter dem künftigen Präsidenten modifiziert werden, die mit Südkorea und Kolumbien anhängigen Verhandlungen über Freihandelsabkommen lehnt er in ihrer jetzigen Form ab und fordert strengere Umweltauflagen ebenso wie höhere Standards bei der Arbeitsplatzsicherheit. ${ }^{32}$ Ob diese Haltung bisher vorübergehendem taktischen Kalkül gegenüber einem - zumal vor dem Hintergrund der aktuellen Finanz- und Wirtschaftskrise - kritischen Kongress geschuldet ist oder tatsächlich seiner Überzeugung entspricht, bleibt abzuwarten; seine positive Grundhaltung zu einer globalisierten Wirtschaft und zum Multilateralismus deutet eigentlich eher darauf hin, dass er ein instinktiver Freihändler ist. Ansonsten jedenfalls könnte er in der laufenden Doha-Runde zu einem für Europäer gar weniger angenehmen Verhandlungspartner werden als McCain es wohl gewesen wäre. Dabei kämen ihm dann sicherlich auch die zunehmenden Zweifel von berufener akademischer Seite an der Freihandelspolitik entgegen (Lawrence Summers, Finanzminister unter Clinton und künftiger Vorsitzender des Nationalen Wirtschaftsrats, Paul A. Samuelson u.a.).

Das Thema Klimawandel schließlich hält Obama zwar für ,eine der größten moralischen Herausforderungen unserer Generation“; 33 ob daraus aber eine neue Führungsrolle der USA in der Klimapolitik entsteht, bleibt abzuwarten. Fest steht, dass er gerade in dieser Frage von den Europäern an seinem Anspruch gemessen wird, Amerikas internationalen Führungsanspruch und seine Glaubwürdigkeit wiederherzustellen. Obama hat angekündigt, sich bei der Weiterentwicklung internationaler Vereinbarungen - etwa bei der VN-Rahmenkonvention zum Klimawandel oder in einem neu zu schaffenden ,Global Energy Forum' (G8-Staaten sowie Brasilien, China, Indien, Mexiko und Südafrika) ${ }^{34}$ - stärker engagieren zu wollen. Er fordert grundsätzlich auch ein System zum Handel mit Emissionsrechten. Allerdings setzt er sich für bindende und durchsetzbare Ziele zur Verringerung der Emissionen auf globaler Ebene auch nur unter der Bedingung einer Einbeziehung Chinas, Indiens und Russlands ein. Lassen sich diese Staaten nicht auf verbindliche Ziele zum Abbau des Treibhausgasausstoßes ein, so werden sich auch die Amerikaner nicht bewegen - zumal auch in diesem Fall der Kongress der eigentliche Adressat europäischer Forderungen bleibt. In jedem Fall ist zu erwarten, dass dieser bei seinen Vorgaben nicht ohne Weiteres den ehrgeizigen Emissionszielen der Europäer folgen wird, den Schadstoffausstoß bis 2020 auf ein Niveau von 20 Prozent unterhalb des Standes von 1990 zu senken. Da sich die generelle Richtung und das Programm doch sehr stark mit dem europäischen Ansatz für mehr Energieeffizienz und Klimaschutz decken - das gilt für die Deckelung der Emissionen wie für die Handelbarkeit von Emissionsrechten - besteht zumindest Hoffnung, dass beide Seiten in der Lage sind, ein Nachfolgekonzept für Kyoto zu entwickeln, welches wie geplant im Dezember 2009 in Kopenhagen beschlossen werden kann.

31 North American Free Trade Agreement.

32 New York Times Online: Democratic Debate in Austin, Tex., Transcript, 21.02.2008, abrufbar unter: http:// www.nytimes.com/2008/02/21/us/politics/21text-demdebate.html?pagewanted=1\&fta=y (letzter Zugriff: 18.12.2008); Bruce Stokes: What's At Stake: Trade, in: National Journal, 02.10.2008.

33 Ken Dilanian: Obama shifts stance on environmental issues, in: USA Today, 18.07.2008.

34 Rudolf: Amerikas neuer globaler Führungsanspruch, 2008, S. 5. 


\section{Perspektiven}

So viel steht fest: Am Ende hat die Wirtschaft die Irak-Politik in diesem Wahlkampf geschlagen; Krieg und Frieden spielten bestenfalls die zweite Rolle. Obama versuchte vor allem mit Wirtschaftsthemen zu punkten. Der Grund liegt auf der Hand: Die Folgen der Immobilien- und Kreditkrise werden das Land noch lange erschüttern. Der Rettung der Immobilienfinanzierer Freddie Mac und Fannie Mae durch den Staat mit etwa 300 Milliarden US-Dollar folgten zunächst die Insolvenz der Investmentbank Lehman Brothers und schließlich die Rettung von ,Wall Street' mit einem Hilfspaket für das Finanzsystem von anfänglich 700 Milliarden US-Dollar. Mittlerweile wird in Washington von Ausgabenprogrammen zur Ankurbelung der Wirtschaft in fast der gleichen Höhe gesprochen; unter 500 Milliarden US-Dollar will unter den Demokraten im Kongress kaum jemand anfangen. Die negativen Kräfte des Abschwungs sind weiter am Werke und deuten auf eine möglicherweise tiefe und lange Rezession hin: die Automobilbranche steht vor dramatischen Einbrüchen, der Einzelhandel stagniert, die Immobilienkrise droht mittlerweile auch Kreditkartennutzer zu erfassen, weitere Preisanstiege sind nach vorübergehender Konsolidierung nicht auszuschließen und die Arbeitslosenrate ist so hoch wie seit 14 Jahren nicht mehr.

Obama wird sich daher zunächst auf die inneramerikanischen Probleme konzentrieren. Dies lässt zwar erwarten, dass das Land sich nicht in große außenpolitische Abenteuer stürzen wird, für die ihm auch die Mittel fehlen. Es birgt aber auch die Gefahr, dass er es versäumt, den Sympathiebonus, den er zurzeit in der Welt genießt, für eine Neubegründung der Stellung Amerikas zu nutzen und manche Hoffungen 2009 Ernüchterung auch im transatlantischen Verhältnis weichen. Wie auch immer: eine Erholung der Wirtschaft ist dringend erforderlich; sie ist auch die Voraussetzung für eine kraftvolle Außenpolitik. Ob der Krise mit wiederentdeckten Konjunkturprogrammen á la Keynes und sozialpolitischen Umverteilungsideen wie zu Zeiten der ,Great Society ' beizukommen ist, ist fraglich. Ein gewaltiges Budgetdefizit und die riesige Staatsverschuldung schränken jedenfalls den fiskalpolitischen Handlungsspielraum erheblich ein. Hinzu kommt, dass im Zeitalter der Globalisierung und angesichts der enormen China-Verschuldung Washington diese Probleme jedenfalls nicht komplett im Alleingang wird bewältigen können.

Davon unbenommen warnt Obama mit seinem nachfrageorientierten Kurs vor einer Eskalation der Ungleichheit und verweist darauf, dass die Mittelklasse der USA erodiert. Er setzt auf langfristige Steuererleichterungen für 95 Prozent aller Familien, zielt dabei aber auf die Durchschnittsverdiener, indem er zunächst eine Steuergutschrift von 1.000 US-Dollar für Haushalte der Mittelklasse und Erleichterungen für Rentner mit einem jährlichen Einkommen unter 50.000 US-Dollar anbietet. Im Gegenzug plant er eine Anhebung der Steuern für Spitzenverdiener mit Bezügen von mehr als 250.000 US-Dollar jährlich und kündigt eine Anhebung der Kapitalgewinnsteuer an. Darüber hinaus plant er aufwendige Infrastrukturprojekte, Investitionen im Energie- und Bildungsbereich. Die Frage nach der Gegenfinanzierung dieser Programme bleibt bislang unbeantwortet. Zwar geht der Internationale Währungsfonds (IWF) davon aus, dass die USA weiteren Spielraum für Konjunkturspritzen haben; doch dies nur, wenn gleichzeitig die mittel- und langfristigen Folgen zusätzlicher Schulden berïcksichtigt werden - wonach es derzeit in Washington nicht aussieht. Das Defizit dürfte im laufenden Haushaltsjahr auf ein Rekorddefizit von 1.2 Billionen US-Dollar beziehungsweise 8,3 Prozent der Wirtschaftskraft des Landes steigen. In Washington aber setzen viele dennoch weiterhin auf eine Politik des billigen Geldes. Ob allerdings allein eine Verminderung der Grenzsteuersätze in dieser Phase, wie pauschal von Republikanern gefordert, die Wirtschaftsaktivität im Lande eher stärken kann, ist ebenso fragwürdig. Wahr- 
scheinlich ist die Kombination aus beidem der richtige Ansatz. Jedenfalls reichen momentan geldpolitische Instrumente allein nicht aus. Die USA und die Welt insgesamt befinden sich tatsächlich in einer Keynesianischen Situation, in der gezielte nachhaltige Konjunkturprogramme angesagt sind; wichtig ist dabei, Geld nicht in Maßnahmen mit extrem kurzfristiger Wirkung zu pumpen, da sie allzu schnell verpuffen. Daneben täte die künftige Administration wohl gut daran, sich so schnell wie möglich um eine gezielte Restrukturierung der Hypotheken beziehungsweise Schulden der privaten Haushalte zu kümmern und auf eine Stärkung der IWF-Fonds mit geringer Konditionalität hinzuwirken, um so ein weiteres Abgleiten der Weltwirtschaft zu verhindern.

Ganz abgesehen davon aber, dass die Handlungsfähigkeit der künftigen Administration auf der Weltbühne schon aus diesen Gründen vorerst erheblich eingeschränkt ist, sollten sich Europäer darüber im Klaren sein, dass zwar einiges im transatlantischen Verhältnis besser werden mag, dass es aber auch unter Obama nicht mehr das sein wird, was es einmal war. Nicht erst seit Bush, sondern seit Ende des Ost-West-Konflikts befinden sich die Beziehungen zwischen Europäischer Union und USA in einem tief greifenden Umbruch; daran ändert auch ein Amtswechsel nichts. Es gibt in vielen Punkten nicht unüberbrückbare, aber doch deutliche Differenzen, die auch mit unterschiedlichen Wertvorstellungen zu tun haben eben ökonomische, geostrategische (insbesondere bezüglich des Aktionsradius), aber auch im weitesten Sinne kulturelle. ${ }^{35}$ An Amerikas Grundüberzeugung, wonach es aufgrund seiner überragenden Machtposition die internationale Ordnung gestalten kann, wird sich auch künftig nichts ändern. Die USA werden auch künftig selektiv, und unter Abwägung ihrer ordnungspolitischen Ziele und Interessen, über den Einsatz ihrer überragenden Mittel in der Welt und mögliche Bündnispartner im Sinne ,flexibler Koalitionen ' entscheiden. Die Hoffnung besteht allerdings, dass unter Obama die liberale Tradition nicht wie in der Vergangenheit als Rechtfertigung einer Interessen- und Machtpolitik dient, die das Ziel des Schutzes der inneren Freiheit lediglich zum Ziel der Stärkung der äußeren Macht des Staates instrumentalisiert. Eben diese Einschätzung kollidierte in den vergangenen Jahren fundamental mit Europas historisch bedingter Auffassung, wonach aus der viel beschworenen gemeinsamen liberalen Grundlage eher die Notwendigkeit vom Rechtsprimat und von der Kooperation auch mit Nichtdemokraten abzuleiten ist. Möglichkeiten für eine größere Einwirkung der Europäischen Union auf die Positionsbestimmung der künftigen Administration dürfte es insofern allemal geben.

35 Vgl. Jens van Scherpenberg: Trade Is No Superglue: The Changing Political Economy of Transatlantic Relations, in: Jeffrey Anderson/John Ikenberry/Thomas Risse (Hrsg.): The End of the West. Crisis and Change in the Atlantic Order, New York/London 2008, S. 127-156; Michael Byers: Crisis, What Crisis? Transatlantic Differences and the Foundations of International Law, in: Jeffrey Anderson/John Ikenberry/Thomas Risse (Hrsg.): The End of the West. Crisis and Change in the Atlantic Order, New York/London 2008, S. 186-209; Jeffrey Anderson: The Sovereign Foundations of Transatlantic Crisis in the Post-9/11 Era, in: Jeffrey Anderson/John Ikenberry/Thomas Risse (Hrsg.): The End of the West. Crisis and Change in the Atlantic Order, New York/ London 2008, S. 210-228; John A. Hall: Passion with Reason, in: Jeffrey Anderson/John Ikenberry/Thomas Risse (Hrsg.): The End of the West. Crisis and Change in the Atlantic Order, New York/London 2008, S. 229246; Thomas Risse: The End of the West? Conclusions, in: Jeffrey Anderson/John Ikenberry/Thomas Risse (Hrsg.): The End of the West. Crisis and Change in the Atlantic Order, New York/London 2008, S. 263-290. 